Supporting Information for

\title{
Effect of Ammonium Salts on the Decarboxylation of Oxaloacetic Acid in Atmospheric Particles
}

Alexandra L. Klodt, Kimberly Zhang, Michael W. Olsen, Jorge L. Fernandez, Filipp Furche, and Sergey A. Nizkorodov Department of Chemistry, University of California, Irvine, CA 92697, USA

\section{Contents}

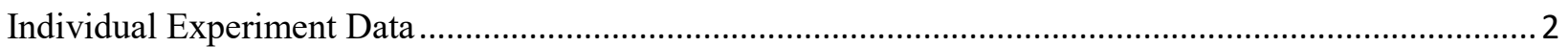

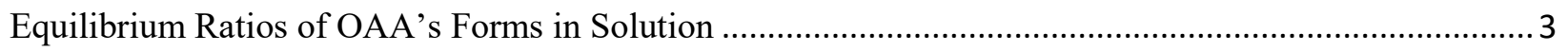

Sample Kinetics Fits for Decarboxylation Reaction ......................................................................... 5

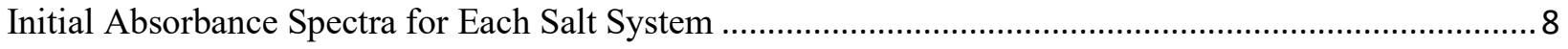

Coordinate Files for Optimized Geometries of Deprotonated Imine ....................................................... 10

Coordinate Files for Optimized Geometries of Deprotonated Enamine …............................................. 11

Coordinate Files for Optimized Geometries of Catalyzed Decarboxylation Transition State...................12

Coordinate Files for Optimized Geometries of Catalyzed Decarboxylation Intermediate .........................13

Coordinate Files for Optimized Geometries of Catalyzed Decarboxylation Product.................................14

Coordinate Files for Optimized Geometries of Deprotonated Oxaloacetic Acid .....................................15

Coordinate Files for Optimized Geometries of Uncatalyzed Decarboxylation Transition State ................16

Coordinate Files for Optimized Geometries of Uncatalyzed Decarboxylation Intermediate .....................17

Coordinate Files for Optimized Geometries of Uncatalyzed Decarboxylation Product...........................18

Coordinate Files for Optimized Geometries of Carbon Dioxide …........................................................19

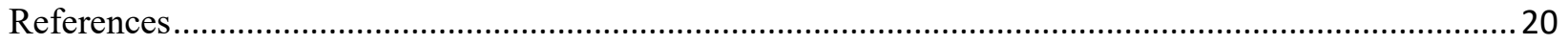




\section{Individual Experiment Data}

Table S1. Data from individual decarboxylation experiments: type of salt added to experimental solution, the concentration of salt added, measured solution $\mathrm{pH}$, calculated rate constant from pseudo-first-order fit (as well as the fit's standard deviation), lifetime of OAA in this solution, and branching ratio to compare lifetime of OAA with respect to $\mathrm{OH}$-oxidation and decarboxylation. The concentration of OAA was approximately $1 \mathrm{mM}$ in all solutions. The

branching ratio is calculated as $Q=\frac{\tau_{d}}{\tau_{O H}}=\frac{k_{O H}[O H]}{k_{d}}$, where $t_{d}$ is the lifetime with respect to decarboxylation and $\mathrm{kOH}_{\mathrm{OH}}$ is the rate constant for reaction with $\mathrm{OH}$, so smaller $(\mathrm{Q}<1)$ values suggest decarboxylation is the faster process for those conditions. Branching ratios are calculated at $\mathrm{pH} 3.7$ for all conditions except solutions containing ammonium bisulfate, which are calculated at $\mathrm{pH} 1$.

\begin{tabular}{|c|c|c|c|c|c|}
\hline Salt Added & $\begin{array}{l}\text { Salt Concentration } \\
\text { (M) }\end{array}$ & $\mathbf{p H}$ & $\begin{array}{l}\text { Rate Constant } \pm 1 \\
\text { Standard Deviation } \\
\left(\mathrm{s}^{-1}\right)\end{array}$ & $\begin{array}{l}\text { Lifetime } \\
\text { (h) }\end{array}$ & $\begin{array}{c}\text { Branching } \\
\text { Ratio (Q) }\end{array}$ \\
\hline None & 0 & & $(4.57 \pm 0.01) \times 10^{-5}$ & 6.1 & $6.53 \times 10^{-3}$ \\
\hline None & 0 & 3.1 & $(5.91 \pm 0.15) \times 10^{-5}$ & 4.7 & $5.05 \times 10^{-3}$ \\
\hline$\left(\mathrm{NH}_{4}\right)_{2} \mathrm{SO}_{4}$ & 0.10 & & $(8.75 \pm 0.03) \times 10^{-5}$ & 3.2 & $3.41 \times 10^{-3}$ \\
\hline$\left(\mathrm{NH}_{4}\right)_{2} \mathrm{SO}_{4}$ & 0.25 & 3.9 & $(8.19 \pm 0.10) \times 10^{-5}$ & 3.4 & $3.65 \times 10^{-3}$ \\
\hline$\left(\mathrm{NH}_{4}\right)_{2} \mathrm{SO}_{4}$ & 0.50 & 3.9 & $(1.24 \pm 0.02) \times 10^{-4}$ & 2.2 & $2.40 \times 10^{-3}$ \\
\hline$\left(\mathrm{NH}_{4}\right)_{2} \mathrm{SO}_{4}$ & 0.50 & & $(1.54 \pm 0.01) \times 10^{-4}$ & 1.8 & $1.94 \times 10^{-3}$ \\
\hline$\left(\mathrm{NH}_{4}\right)_{2} \mathrm{SO}_{4}$ & 0.75 & 3.5 & $(1.45 \pm 0.02) \times 10^{-4}$ & 1.9 & $2.06 \times 10^{-3}$ \\
\hline$\left(\mathrm{NH}_{4}\right)_{2} \mathrm{SO}_{4}$ & 1.0 & 4.0 & $(1.86 \pm 0.01) \times 10^{-4}$ & 1.5 & $1.60 \times 10^{-3}$ \\
\hline$\left(\mathrm{NH}_{4}\right)_{2} \mathrm{SO}_{4}$ & 1.0 & 4.0 & $(1.89 \pm 0.02) \times 10^{-4}$ & 1.5 & $1.58 \times 10^{-3}$ \\
\hline$\left(\mathrm{NH}_{4}\right)_{2} \mathrm{SO}_{4}$ & 1.35 & 3.5 & $(2.54 \pm 0.01) \times 10^{-4}$ & 1.1 & $1.17 \times 10^{-3}$ \\
\hline$\left(\mathrm{NH}_{4}\right)_{2} \mathrm{SO}_{4}$ & 1.48 & 3.7 & $(2.52 \pm 0.02) \times 10^{-4}$ & 1.1 & $1.19 \times 10^{-3}$ \\
\hline$\left(\mathrm{NH}_{4}\right)_{2} \mathrm{SO}_{4}$ & 1.5 & 4.0 & $(2.39 \pm 0.04) \times 10^{-4}$ & 1.2 & $1.25 \times 10^{-3}$ \\
\hline$\left(\mathrm{NH}_{4}\right)_{2} \mathrm{SO}_{4}$ & 1.85 & 3.8 & $(2.53 \pm 0.02) \times 10^{-4}$ & 1.1 & $1.18 \times 10^{-3}$ \\
\hline$\left(\mathrm{NH}_{4}\right)_{2} \mathrm{SO}_{4}$ & 2.0 & 4.0 & $(2.70 \pm 0.03) \times 10^{-4}$ & 1.0 & $1.10 \times 10^{-3}$ \\
\hline$\left(\mathrm{NH}_{4}\right)_{2} \mathrm{SO}_{4}$ & 2.25 & 3.9 & $(2.63 \pm 0.02) \times 10^{-4}$ & 1.1 & $1.13 \times 10^{-3}$ \\
\hline
\end{tabular}




\begin{tabular}{cccccc|}
\hline $\left.\mathbf{N H}_{4}\right)_{2} \mathbf{S O}_{4}$ & 2.5 & 4.0 & $(2.32 \pm 0.01) \times 10^{-4}$ & 1.2 & $1.29 \times 10^{-3}$ \\
\hline $\mathbf{N H}_{4} \mathbf{C l}$ & 0.25 & 3.1 & $(6.92 \pm 0.12) \times 10^{-5}$ & 4.0 & $4.31 \times 10^{-3}$ \\
\hline $\mathbf{N H}_{4} \mathbf{C l}$ & 0.5 & 3.0 & $(7.38 \pm 0.10) \times 10^{-5}$ & 3.8 & $4.04 \times 10^{-3}$ \\
\hline $\mathbf{N H}_{4} \mathbf{C l}$ & 1.0 & 3.0 & $(9.11 \pm 0.16) \times 10^{-5}$ & 3.1 & $3.28 \times 10^{-3}$ \\
\hline $\mathbf{N H}_{4} \mathbf{C l}$ & 1.5 & 2.9 & $(1.57 \pm 0.01) \times 10^{-4}$ & 1.8 & $1.90 \times 10^{-3}$ \\
\hline $\mathbf{N H}_{4} \mathbf{C l}$ & 2.0 & 2.9 & $(1.97 \pm 0.09) \times 10^{-4}$ & 1.4 & $1.51 \times 10^{-3}$ \\
\hline $\mathbf{N H}_{4} \mathbf{C l}$ & 2.5 & 2.7 & $(2.35 \pm 0.01) \times 10^{-4}$ & 1.2 & $1.27 \times 10^{-3}$ \\
\hline $\mathbf{N H}_{4} \mathrm{HSO}_{4}$ & 0.5 & 1.3 & $(8.05 \pm 0.12) \times 10^{-6}$ & 35.5 & $4.23 \times 10^{-2}$ \\
\hline $\mathbf{N H}_{4} \mathbf{H S O}_{4}$ & 0.8 & 1.0 & $(6.15 \pm 0.10) \times 10^{-6}$ & 45.2 & $5.54 \times 10^{-2}$ \\
\hline $\mathbf{N H}_{4} \mathrm{HSO}_{4}$ & 1.5 & 0.4 & $(4.49 \pm 0.03) \times 10^{-6}$ & 62.0 & $7.60 \times 10^{-2}$ \\
\hline $\mathbf{N H}_{4} \mathrm{HSO}_{4}$ & 2.0 & 0.2 & $(2.37 \pm 0.04) \times 10^{-6}$ & 117 & $1.44 \times 10^{-1}$ \\
\hline $\mathbf{H}_{2} \mathbf{S O}_{4}$ & 0 & 1.0 & $(4.36 \pm 0.04) \times 10^{-6}$ & 63.7 & $7.82 \times 10^{-2}$ \\
\hline $\mathbf{N a}_{2} \mathbf{S O}_{4}$ & 0.25 & 3.5 & $(5.83 \pm 0.05) \times 10^{-5}$ & 4.8 & $5.11 \times 10^{-3}$ \\
\hline $\mathbf{N a}_{2} \mathbf{S O}_{4}$ & 0.75 & 3.4 & $(3.55 \pm 0.10) \times 10^{-5}$ & 7.8 & $8.39 \times 10^{-3}$ \\
\hline $\mathbf{N a}_{2} \mathbf{S O}_{4}$ & 1.0 & 3.4 & $(5.44 \pm 0.04) \times 10^{-5}$ & 5.1 & $5.48 \times 10^{-3}$ \\
\hline $\mathbf{N a}_{2} \mathbf{S O}_{4}$ & 1.34 & 3.6 & $(4.94 \pm 0.03) \times 10^{-5}$ & 5.6 & $6.03 \times 10^{-3}$ \\
\hline
\end{tabular}

\section{Equilibrium Ratios of OAA's Forms in Solution}

Table S2. The possible forms OAA takes in solution and the abundances as determined by Kozlowski et al. ${ }^{1}$

\begin{tabular}{|c|l|l|}
\hline \multicolumn{1}{|c|}{ Structure } & pH 3.7 (\%) & pH 1 (\%) \\
\hline & 0 & 3.4 \\
\hline
\end{tabular}




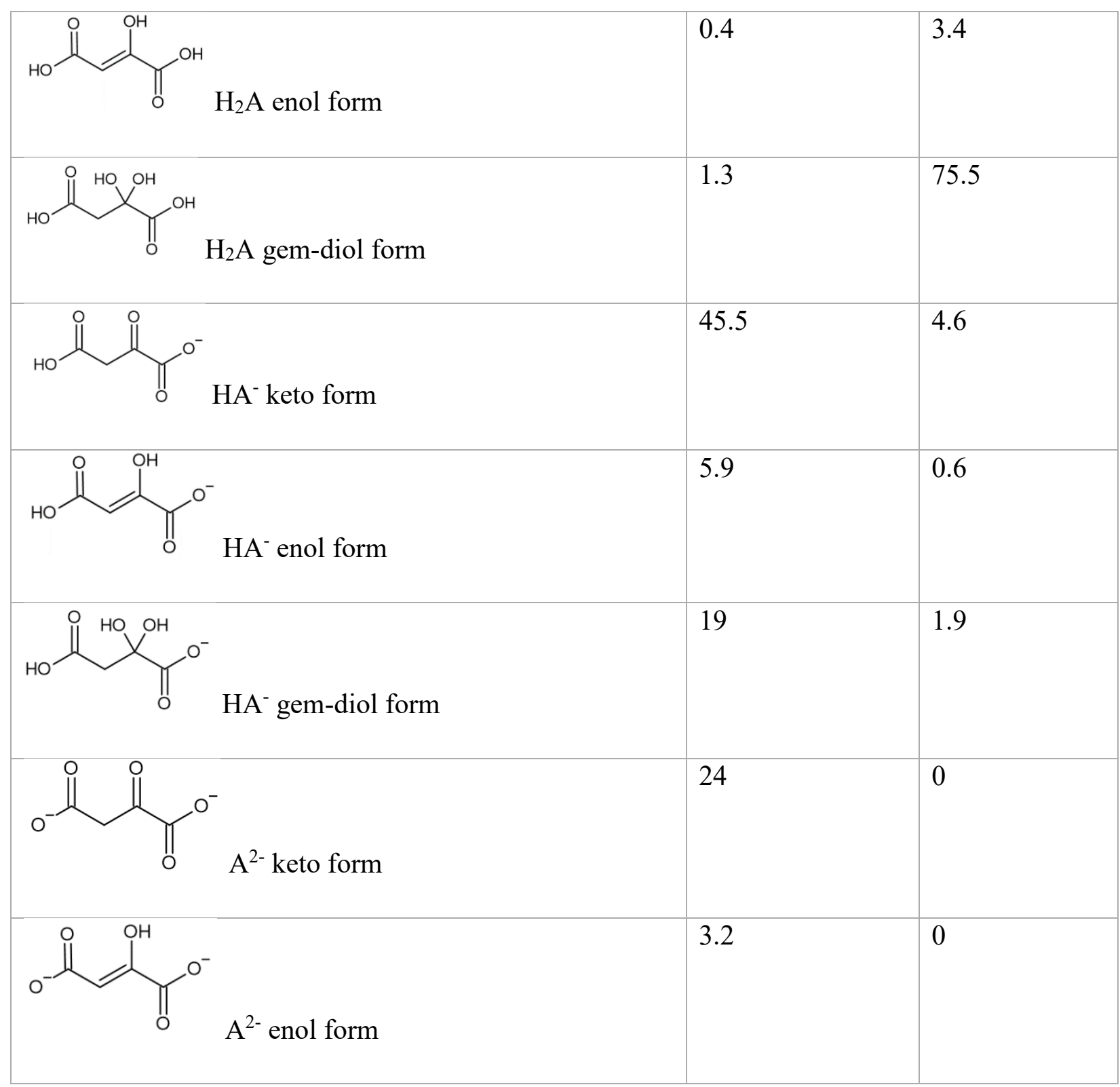

Table S3. Values used to calculate $\mathrm{OH}$ rate constants. The protonated keto form could not be included in the calculation of the $\mathrm{OH}$-oxidation rate constants, so values from Table S2 were renormalized to exclude it from the calculation. We do not expect this to have a significant effect on the calculated $\mathrm{OH}$-oxidation rate as the keto forms generally react an order of magnitude more slowly than the gem-diol forms, ${ }^{2}$ and the protonated keto form only accounts for $3.4 \%$ of the abundance at $\mathrm{pH} 1$ and is not present at $\mathrm{pH} 3.7 .{ }^{1} \mathrm{OH}$-oxidation rate constants for the enol forms of OAA were also not calculable using the SAR. ${ }^{3,4}$ However, but-2-enedioic acid, which 
has the same structure as enol OAA with the exception of enol OAA's vinylic alcohol group, reacts quickly with the $\mathrm{OH}$ radical $\left(6 \times 10^{9} \mathrm{M}^{-1} \mathrm{~s}^{-1}\right.$; see fumaric acid and maleic acid in Buxton et al. ${ }^{5}$ ). Additionally, but-2-enedioic acid only has two equivalent hydrogens which may be abstracted by $\mathrm{OH}$, so the reactivity of each hydrogen should be half of but-2-enedioic acid's total $\mathrm{OH}$ reactivity, or $3 \times 10^{9} \mathrm{M}^{-1} \mathrm{~s}^{-1}$. We therefore assumed the $\mathrm{OH}$ reactivity of enol OAA's vinylic hydrogen to be equivalent to the reactivity of one of but-2-enedioic acid's vinylic hydrogens and the $\mathrm{OH}$ reactivity of enol OAA's vinylic alcohol group to be $6.9 \times 10^{7} \mathrm{M}^{-1} \mathrm{~s}^{-1}$, which is the base value for alcohol groups in the SAR. ${ }^{3}$

\begin{tabular}{|c|c|c|c|}
\hline Form & $\begin{array}{l}\text { OH-oxidation rate } \\
\text { constant }\left(\mathbf{M}^{-1} \mathbf{s}^{-1}\right)\end{array}$ & $\begin{array}{c}\text { Ratio used for pH } 3.7 \\
(\%)\end{array}$ & $\begin{array}{c}\text { Ratio used for } \mathrm{pH} 1 \\
(\%)\end{array}$ \\
\hline $\mathrm{H}_{2} \mathrm{~A}$ keto form & $7.01 \times 10^{6}$ & 0.2 & 11.0 \\
\hline $\mathrm{H}_{2} \mathrm{~A}$ enol form & $3.07 \times 10^{9}$ & 0.4 & 3.5 \\
\hline $\mathrm{H}_{2} \mathrm{~A}$ gem-diol form & $2.61 \times 10^{8}$ & 1.3 & 78.2 \\
\hline $\mathrm{HA}^{-}$keto form & $1.23 \times 10^{7}$ & 45.5 & 4.8 \\
\hline $\mathrm{HA}^{-}$enol form & $3.07 \times 10^{9}$ & 5.9 & 0.6 \\
\hline $\mathrm{HA}^{-}$gem-diol form & $4.51 \times 10^{8}$ & 19.0 & 2.0 \\
\hline $\mathrm{A}^{2-}$ keto form & $4.22 \times 10^{7}$ & 24 & 0 \\
\hline $\mathrm{A}^{2-}$ enol form & $3.07 \times 10^{9}$ & 3.2 & 0 \\
\hline $\begin{array}{l}\text { Final rate constant } \\
\text { for OH oxidation }\end{array}$ & & $2.98 \times 10^{8} \mathrm{M}^{-1} \mathrm{~s}^{-1}$ & $3.41 \times 10^{8} \mathrm{M}^{-1} \mathrm{~s}^{-1}$ \\
\hline
\end{tabular}

\section{Sample Kinetics Fits for Decarboxylation Reaction}

When monitoring the decarboxylation of OAA by UV-Vis, it can be seen (Figures S1-S3) that the decrease at $260 \mathrm{~nm}$ is not strictly a first-order decay until after some time has passed, corresponding to the keto/enol and keto/gem-diol conversions both reaching equilibrium. Since the conversion between OAA's keto and gem-diol forms is faster in water than between the keto and enol forms, ${ }^{6}$ it is likely the keto/enol interconversion that controls the change in the preequilibrium absorbance. However, to the best of our knowledge, the rate of keto/enol interconversion has not been determined at $\mathrm{pH}$ values smaller than 5 (although the keto/enol 
equilibrium ratios are available $\left.{ }^{1}\right)$. At $\mathrm{pH}$ values above 5 , the rate has been shown to be highly $\mathrm{pH}$ dependent, ${ }^{6}$ so it would not be reasonable to apply rate constants obtained at higher $\mathrm{pH}$ values to our data. In any case, the expected first-order kinetics are observed after the solution has had time to reach keto/enol equilibrium. To avoid interference from the solution equilibration, we began fitting once the data had adopted a first-order decay pattern. The first point for the fit was chosen by performing sample fits beginning at each data point, then using the one with the earliest starting point with which the later fits agree within the standard deviation of the chosen fit. This difference is small under conditions of high ammonium concentration ( 0 to 5 percent), but much larger with low ammonium concentrations (5 to 10 percent) and/or high acidity (10 to 25 percent).

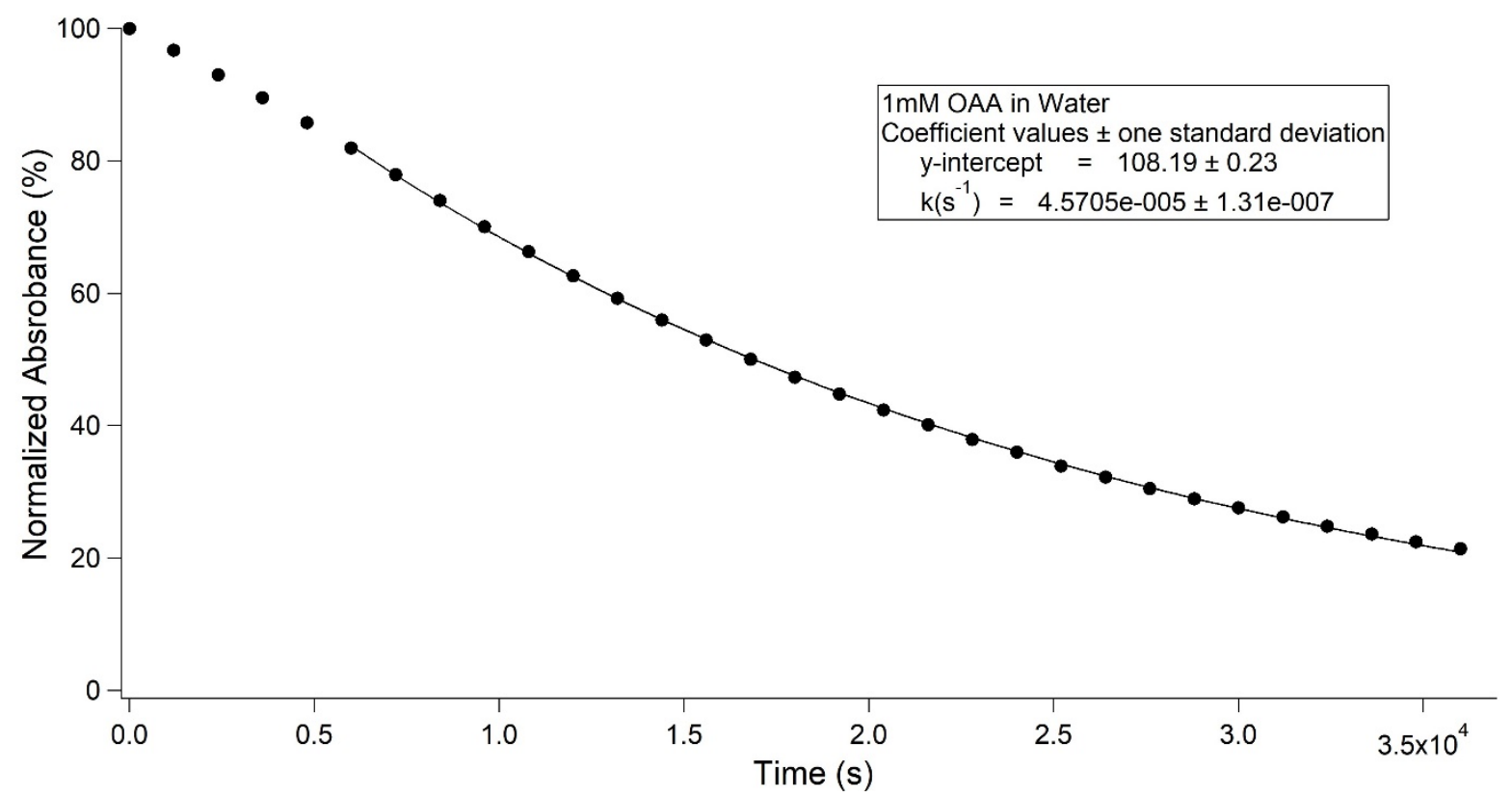

Figure S1. Absorbance at $260 \mathrm{~nm}$, normalized to time 0 , for $1 \mathrm{mM}$ OAA in pure water. The data is shown with individual dots and the fit with a solid line. 


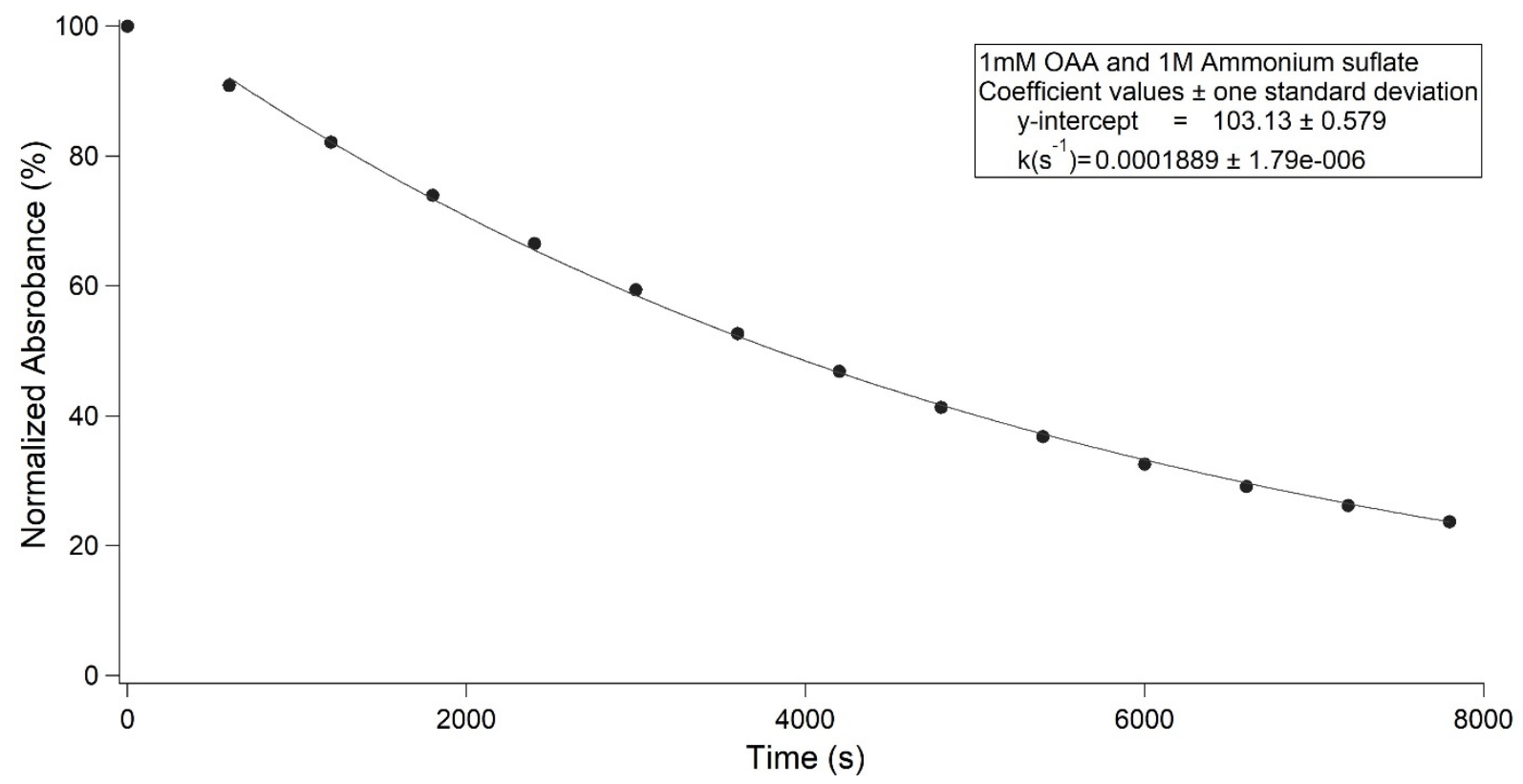

Figure S2. Absorbance at $260 \mathrm{~nm}$, normalized to time 0 , for $1 \mathrm{mM} \mathrm{OAA}$ in $1 \mathrm{M}$ ammonium sulfate solution. The data is shown with individual dots and the fit with a solid line.

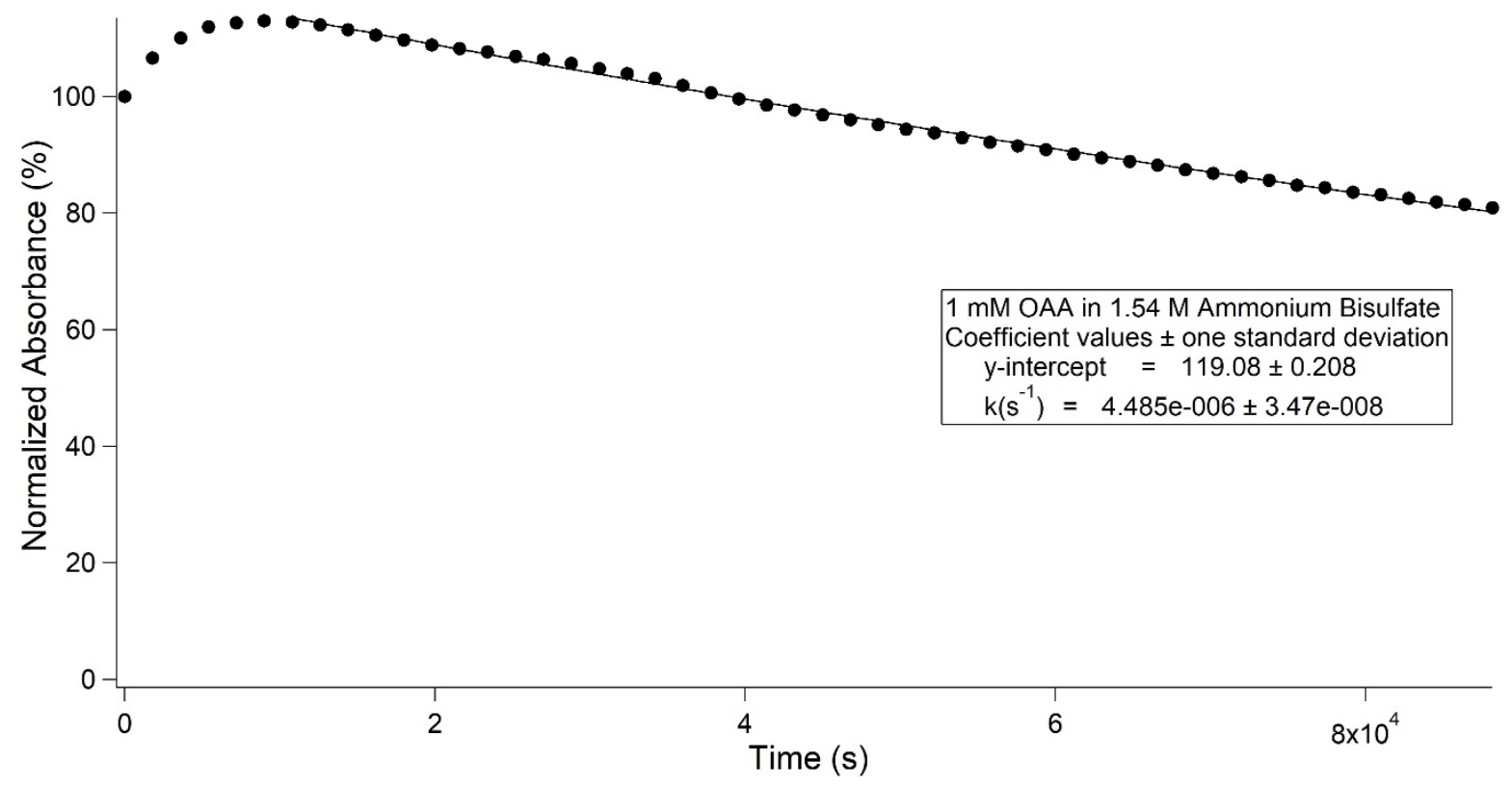

Figure S3. Absorbance at $260 \mathrm{~nm}$, normalized to time 0 , for $1 \mathrm{mM}$ OAA in $1.54 \mathrm{M}$ ammonium bisulfate solution. The data is shown with individual dots and the fit with a solid line. 


\section{Initial Absorbance Spectra for Each Salt System}

Although all solutions were prepared at close to $1 \mathrm{mM}$ concentrations of OAA as possible, the actual concentration of each solution was used to scale each spectrum by $1 \mathrm{mM} /($ exact concentration) to make the peak heights more directly comparable in Figures below.

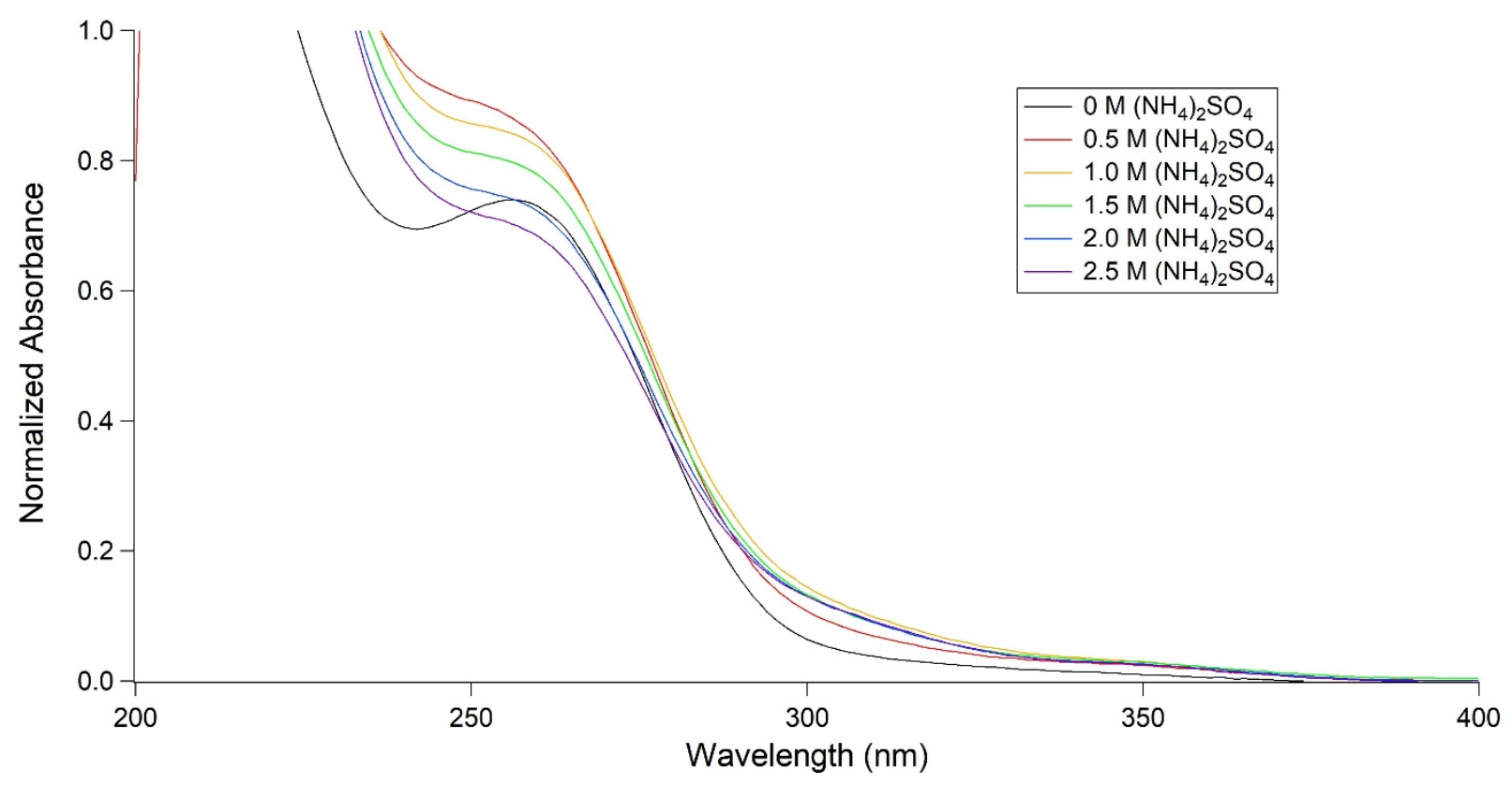

Figure S4. Initial absorbance spectra for solutions containing ammonium sulfate.

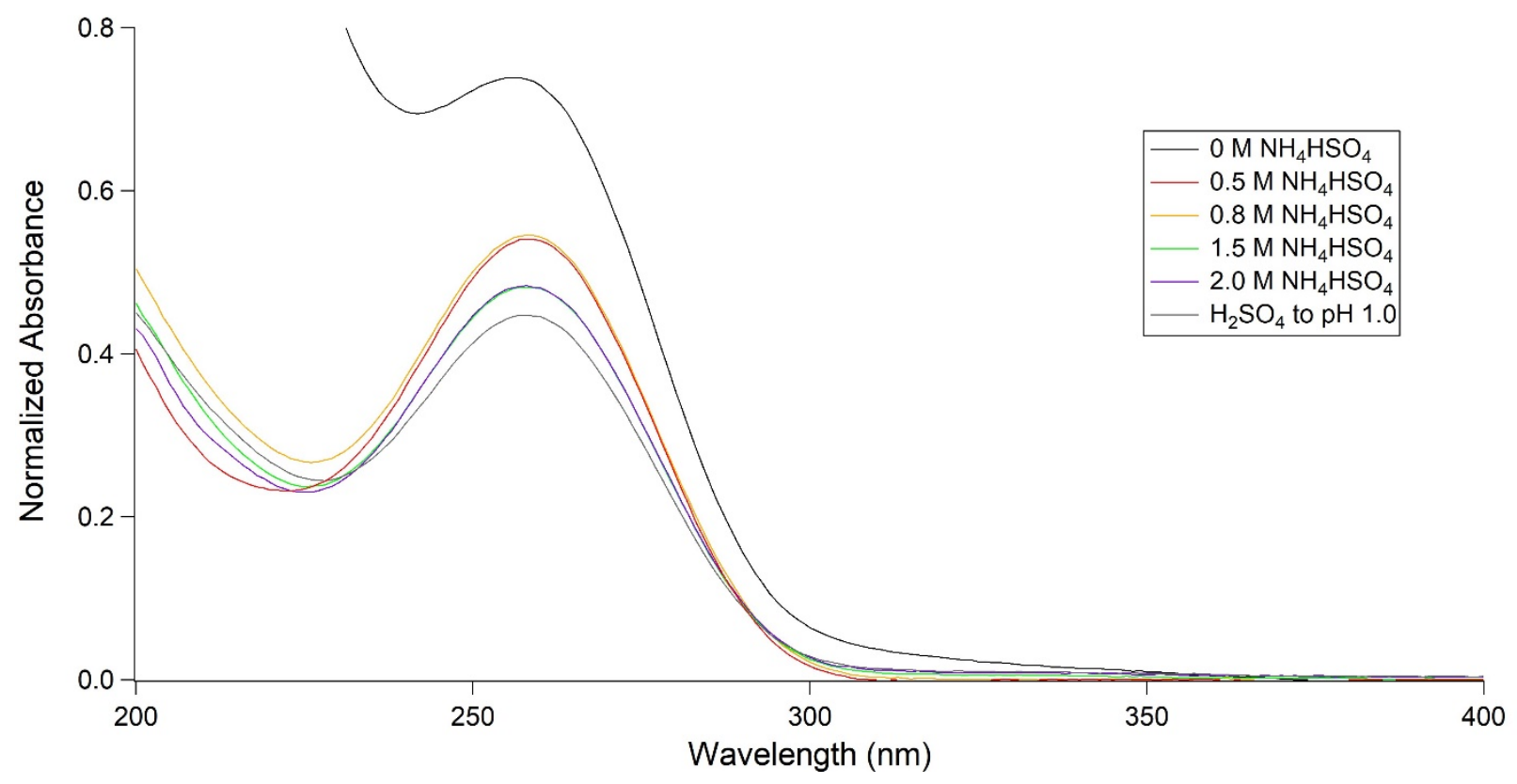


Figure S5. Absorbance spectra for OAA in ammonium bisulfate at their maximum value.

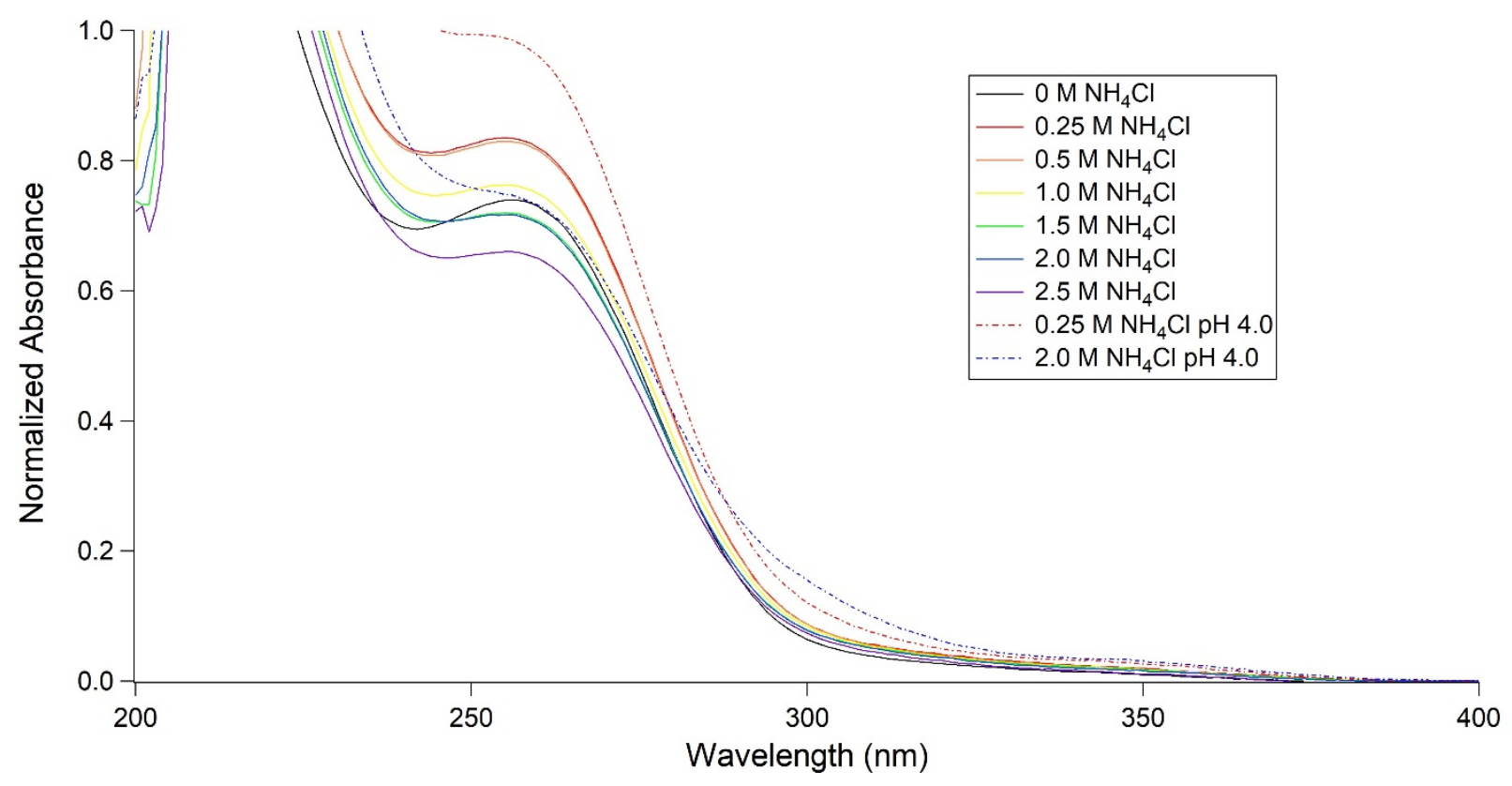

Figure S6. Initial absorbance spectra for solutions containing ammonium chloride. The traces in dashed lines have the $\mathrm{pH}$ adjusted to 4.0 so that the enol band is present as in the ammonium sulfate conditions.

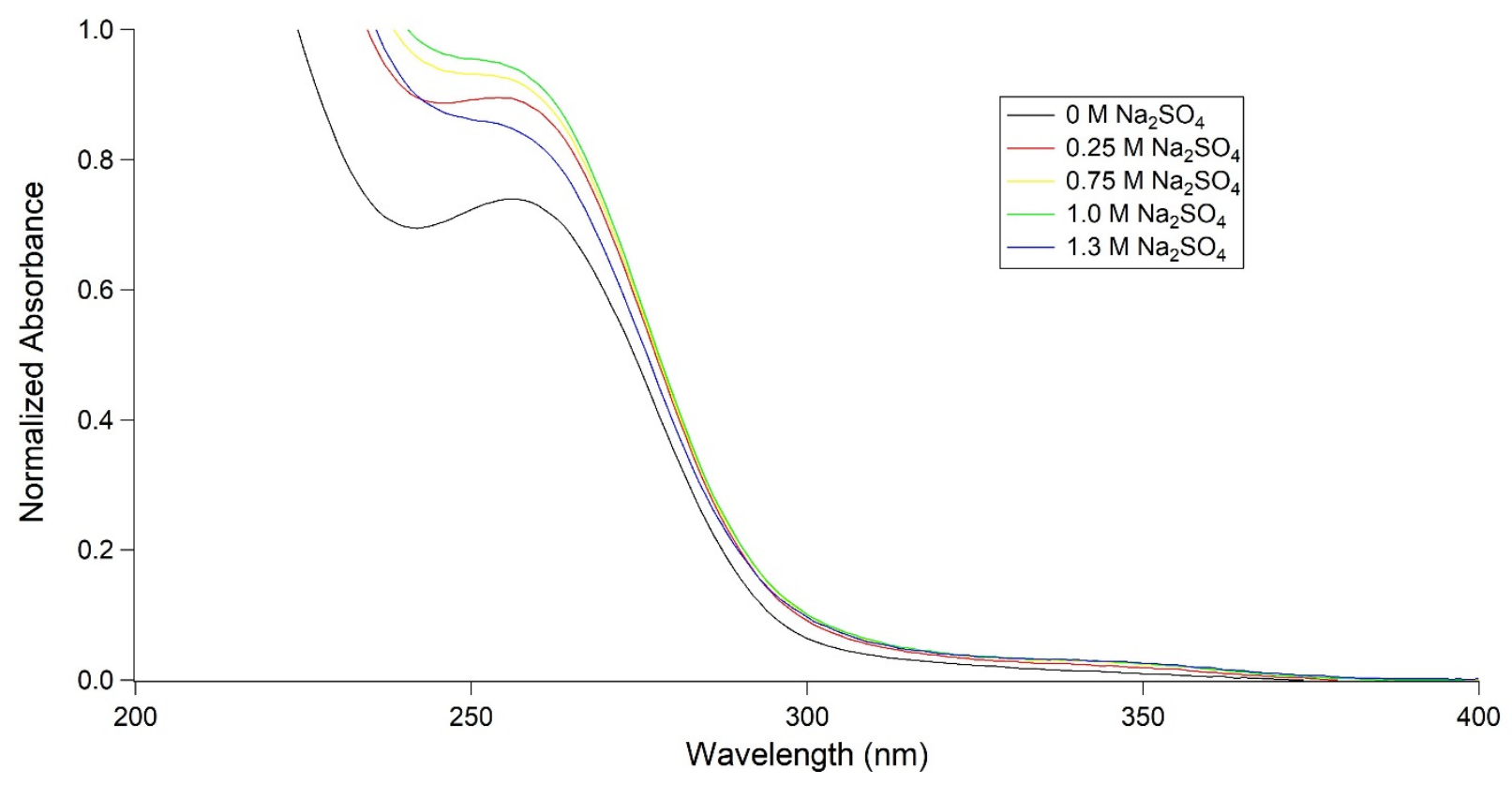

Figure S7. Initial absorbance spectra for solutions containing sodium sulfate. 
Coordinate Files for Optimized Geometries of Deprotonated Imine<smiles>N=C(CC(=O)O)C(=O)[O-]</smiles>

\section{Without COSMO}

$\begin{array}{lrrrl}\text { Senergy SCF } & \text { SCFKIN } & \text { SCFPOT } & \\ 17-510.3349531413 & 508.2915145735 & -1018.626467715 & \\ \text { \$coord } & & & \\ -4.78515425662849 & -0.74763417640674 & -0.03163919229291 & \mathrm{c} \\ -2.29291991911946 & 0.76900291748500 & -0.01420992174409 & \mathrm{c} \\ 0.14524943365906 & -0.65639863840006 & -0.03028707484980 & \mathrm{c} \\ 2.59261394688963 & 0.83141153945583 & -0.02457943271809 & \mathrm{c} \\ 0.18028088307231 & -1.91646002091955 & -1.66689517115228 & \mathrm{~h} \\ 0.18789662013595 & -1.93728974107206 & 1.59028762542637 & \mathrm{~h} \\ -2.31527212246562 & 3.16291382864154 & 0.01427597658222 & \mathrm{n} \\ -6.74533269599123 & 0.55647464646568 & -0.02216958867767 & \mathrm{o} \\ -4.57080946090254 & -3.08818960654221 & -0.05278572689850 & \text { o } \\ 2.44382108154830 & 3.31055190140840 & 0.00609641739918 & \mathrm{o} \\ 4.61721802993885 & -0.24397932950426 & -0.04652385769604 & \mathrm{o} \\ 0.56787680066169 & 3.79345418486884 & 0.01742769987252 & \mathrm{~h} \\ -4.13367232012813 & 3.80800600329577 & 0.02159284297382 & \mathrm{~h}\end{array}$

\section{With COSMO}

Senergy SCF

$17-510.3349531413$

SCFKIN SCFPOT

Send

\$coord

$\begin{array}{rrrr}-4.78515425662849 & -0.74763417640674 & -0.03163919229291 & \mathrm{c} \\ -2.29291991911946 & 0.76900291748500 & -0.01420992174409 & \mathrm{c} \\ 0.14524943365906 & -0.65639863840006 & -0.03028707484980 & \mathrm{c} \\ 2.59261394688963 & 0.83141153945583 & -0.02457943271809 & \mathrm{c} \\ 0.18028088307231 & -1.91646002091955 & -1.66689517115228 & \mathrm{~h} \\ 0.18789662013595 & -1.93728974107206 & 1.59028762542637 & \mathrm{~h} \\ -2.31527212246562 & 3.16291382864154 & 0.01427597658222 & \mathrm{n} \\ -6.74533269599123 & 0.55647464646568 & -0.02216958867767 & \mathrm{o} \\ -4.57080946090254 & -3.08818960654221 & -0.05278572689850 & \text { o } \\ 2.44382108154830 & 3.31055190140840 & 0.00609641739918 & \text { o } \\ 4.61721802993885 & -0.24397932950426 & -0.04652385769604 & \text { o } \\ 0.56787680066169 & 3.79345418486884 & 0.01742769987252 & \mathrm{~h} \\ -4.13367232012813 & 3.80800600329577 & 0.02159284297382 & \mathrm{~h}\end{array}$

\$end 


\section{Coordinate Files for Optimized Geometries of Deprotonated Enamine}<smiles>N/C(=C\C(=O)O)C(=O)[O-]</smiles>

\section{Without COSMO}

\$energy SCF

$1-510.1959462169$

$14-510.2395310178$

Send

\$coord

-6.25582272553272
-3.47297014761418
-1.48700818847376
1.11953742426624
-3.38839674867898
-1.96631339578729
1.65784382168735
2.90210971080549
-6.54773202742642
-7.84213520401772
3.46942376102651
-5.13736337241748
-1.75987342137447

Send

\section{With COSMO}

Senergy SCF

$1-510.1959462169$

$25-510.3431509366$

Send

\$coord

-6.21459817096023
-3.48843926287912
-1.51248005742633
1.11109731901526
-3.38539753939801
-1.98163051667599
1.65126189216568
2.82908308041478
-6.48320564064128
-7.89710873908067
3.46132815406267
-5.05346952796942
-1.74514150416479
Send

$$
\begin{array}{ll}
\text { SCFKIN } & \text { SCFPOT } \\
507.7392400550 & -1017.935186272 \\
508.2800472763 & -1018.519578294
\end{array}
$$

$\begin{array}{ll}-0.47790944158188 & 0.37156797756573 \mathrm{c}\end{array}$ $0.39066987378600-0.13921344165913$ c

$-1.24265002451863 \quad 0.16350502915569 \mathrm{c}$ $-0.72205113147835-0.22902615478086 \mathrm{c}$

$2.79773078243661-0.85999660847036 \mathrm{n}$

$-3.13132008985668 \quad 0.76689265265097 \mathrm{~h}$

$\begin{array}{lll}1.72361874683273 & -1.00258072947519 & \text { o }\end{array}$

$\begin{array}{lll}-2.16350683783555 & 0.03481418541245 \quad \mathrm{o}\end{array}$

$\begin{array}{lll}-2.68735921569896 & 1.05742131104828 & \text { o }\end{array}$

$\begin{array}{lll}1.23660617041641 & 0.00325592767208 & \text { o }\end{array}$

$1.71277947755431-1.16529258617255 \mathrm{~h}$

$3.58157857120686-0.93486881934089 \mathrm{~h}$

$3.67419207930544-1.27015035394861 \mathrm{~h}$
SCFKIN SCFPOT

$507.7392400550 \quad-1017.935186272$

$508.2876771049-1018.630828041$

$-0.50464941400774$

0.41759463786338

$-1.22595369470416$

$-0.71712360008326$

2.82131955086889

$-3.11672288395676$

1.67238341343962

$-2.24173601714510$

$-2.73183744530054$

1.11757703902127

1.78395969871264

3.73248988897620

3.68507778688386
$0.37527493519921 \mathrm{c}$

$-0.14511028633867 \mathrm{c}$

$0.16091367560319 \mathrm{c}$

$-0.23015141484873 \mathrm{c}$

$-0.86532825888788 \mathrm{n}$

$0.76329162512486 \mathrm{~h}$

-0.98515060065886 o

0.06213598504373 o

1.07035592945842 о

0.03606579751249 о

$-1.18653011011987 \mathrm{~h}$

$-0.98583459826963 \mathrm{~h}$

$-1.27360428916062 \mathrm{~h}$ 


\section{Coordinate Files for Optimized Geometries of Catalyzed Decarboxylation Transition State}<smiles>O=C1CC(C(=O)[O-])=C[NH2+]1</smiles>

Without COSMO

Senergy SCF

$1-510.1969052890$

SCFKIN SCFPOT

$58-510.2169666653$

$507.9858345840-1018.182739873$

Send

\$coord

$-3.44199569194660$

$-0.99193041588579$

$508.2289497001-1018.445916365$

0.87659042256370

3.86302014880163

0.42927336507091

1.96432520452999

$-3.81455578889647$

$-4.70400440119252$

$-0.83255813458357$

4.68278247322351

4.22997368187346

$-0.42114867937203$

$-1.03094673842939 \mathrm{c}$

0.13554936588399

$0.51968100723091 \mathrm{c}$

$-1.65092703612057$

$0.98511632797023 \mathrm{c}$

$-0.47140900584468-0.97237856022873 \mathrm{c}$

$-3.54738202817216 \quad 0.37373441114186 \mathrm{~h}$

$-1.48008741170443 \quad 2.71799767115989 \mathrm{~h}$

$\begin{array}{lll}-2.65380149794210 & -1.58328025860197 \text { o }\end{array}$

$\begin{array}{lll}1.52519880330530 & -1.47155019211526 \text { o }\end{array}$

$2.52228916218088 \quad 1.23555280907812 \mathrm{n}$

$\begin{array}{lll}-2.23874966876125 & -2.11463156044942 \quad \text { o }\end{array}$

$-2.20790418838354$

1.70893459802325

-0.43302726063385 o

0.87811125030310

3.58043259047400

$0.40299705012911 \mathrm{~h}$

3.23366982387751

$1.67914173737672 \mathrm{~h}$

Send

\section{With COSMO}

Senergy SCF

$1-510.3142144989$

$21-510.3142599535$

\$end

\$coord

$-3.03427366688651$

$-1.03939457357870$

0.90546224932917

3.69285828077633

2.05094187905678

0.75141914790139

$-2.94656744421013$

$-4.55407910764890$

$-1.32282475657175$

4.21299871542780

4.05129747804315

$-2.68511386709092$

$-0.10928302499998$

\$end
SCFKIN SCFPOT

$508.2321731367 \quad-1018.546387636$

$508.2602631873-1018.574523141$
$-0.76643828474428$

0.96726068396646

$-0.00356986021305$

$-0.29432570295772$

1.24819226439226

$-1.95516626173509$

$-3.04610726525747$

0.32498933115536

3.39310480756612

$-2.47655302982087$

1.80501292306534

3.84081888423959

4.72270745696458
$0.20788879181380 \mathrm{c}$ $-1.02283606918911 \mathrm{c}$ $-2.44755634719791 \mathrm{c}$ $0.44164344456781 \mathrm{c}$ $-3.59722429715666 \mathrm{~h}$ $-3.01437081803248 \mathrm{~h}$ -0.34459969292991 o 1.65002471961029 o $-0.47023784699543 \mathrm{n}$ 0.51127127093896 o 1.14742841362094 o $0.78401570305802 \mathrm{~h}$ $-1.07478144796341 \mathrm{~h}$ 


\section{Coordinate Files for Optimized Geometries of Catalyzed Decarboxylation Intermediate}<smiles>C=C(N)C(=O)[O-]</smiles>

$\begin{array}{lcccc}\text { Senergy SCF } & \text { SCFKIN } & \text { SCFPOT } \\ 1-321.7189111597 & 320.1485782980 & -641.8674894577 & \\ 11-321.7450882603 & 320.4612986593 & -642.2063869196 & \\ \text { \$end } & & & \\ \text { \$coord } & & & \\ -6.06464364938709 & -0.53468554493112 & -0.00000137843277 \mathrm{c} \\ -3.58173048211600 & 1.04273027045942 & -0.00001435628898 \mathrm{c} \\ -1.28068079363292 & -0.02709941714606 & -0.00000511147927 \mathrm{c} \\ -4.08127242637692 & 3.56902225525004 & 0.00000088154673 \mathrm{n} \\ -1.16612945774178 & -2.06467531254580 & -0.00000049324144 \mathrm{~h} \\ 0.44844731675615 & 1.08225101863541 & 0.00000140187489 \mathrm{~h} \\ -8.03301058742519 & 0.78824415348753 & -0.00002060588175 \mathrm{o} \\ -5.84620862958625 & -2.86397015643672 & 0.00001959120385 \mathrm{o} \\ -5.94876491229124 & 3.95981433765945 & 0.00001868495789 \mathrm{~h} \\ -2.73233026199513 & 4.89200851355313 & 0.00000138574086 \mathrm{~h}\end{array}$

Send

\section{With COSMO}

Senergy SCF

$1-321.8354956969$

$10-321.8542154782$

Send

\$coord

-6.03352619679689
-3.61763952141288
-1.31973373927497
-4.07912302038535
-1.18754250542507
0.40281560517282
-8.06904817776008
-5.79979154864590
-5.89504799800915
-2.68768678125887
end

$-0.54877804561190$ 1.05513214557129

$-0.03059710566772$

3.58326764985491

$-2.06619153944276$

1.07998139006151

0.66489761895855

$-2.89435691121703$

4.13173031543696

4.86855460004147
SCFPOT

$-642.0029188393$

$-642.3419829473$
$-0.00011583452509 \mathrm{c}$ $-0.00012676644241 \mathrm{c}$ $-0.00083078884260 \mathrm{c}$ $-0.00049993534892 \mathrm{n}$ $-0.00083922929542 \mathrm{~h}$ $-0.00150354918450 \mathrm{~h}$ -0.00557547909084 о 0.00507340129222 о $0.00265361475757 \mathrm{~h}$ $0.00176456667994 \mathrm{~h}$ 
Coordinate Files for Optimized Geometries of Catalyzed Decarboxylation Product<smiles>CC(=N)C(=O)[O-]</smiles>

$\begin{array}{lcccc}\text { Senergy SCF } & \text { SCFKIN } & \text { SCFPOT } & \\ 1-321.7292603270 & 320.1981841977 & -641.9274445247 & \\ 10-321.7565695832 & 320.4775535500 & -642.2341231333 & \\ \text { Send } & & & & \\ \text { \$coord } & & & \\ -6.00785085947250 & 3.64406453019673 & -0.00675559406776 & \mathrm{c} \\ -3.42463877408778 & 5.05303154702654 & 0.00247983061051 & \mathrm{c} \\ -1.07373895057244 & 3.48265518546369 & 0.00131340310631 \mathrm{c} \\ -1.06591126462617 & 2.22805868149981 & 1.64048218867222 & \mathrm{~h} \\ 0.60080248156940 & 4.68669057906609 & 0.00828731966653 & \mathrm{~h} \\ -1.06015776043284 & 2.23909213891064 & -1.64620509277910 & \mathrm{~h} \\ -3.30669309226137 & 7.44853401048599 & 0.01072556759664 & \mathrm{n} \\ -7.89328205744320 & 5.05422813022262 & -0.00520140432359 & \mathrm{o} \\ -5.87001883863567 & 1.29477027007249 & -0.01451980541862 & \mathrm{o} \\ -5.16757936550019 & 8.01030208872290 & 0.00939358693682 & \mathrm{~h}\end{array}$

Send

With COSMO

Senergy SCF

$1-321.8384766030$

SCFKIN SCFPOT

$\begin{array}{llll}10 & -321.8590884559 & 320.5026825511 & -642.3617710070\end{array}$

Send

\$coord

$-5.98775408372597$

$-3.41666725484977$

$-1.07314833002027$

$-1.04371506076006$

0.59893167380359

320.2166066723
320.5026825511

$-642.0550832753$

$-1.03877028587727$

3.65165076818780

$-0.00675710392156 \mathrm{c}$

$5.03828773035572 \quad 0.00303611847124 \mathrm{c}$

$3.47722689252460 \quad 0.00050021480328 \mathrm{c}$

$2.23198546863177 \quad 1.64618979363850 \mathrm{~h}$

$4.67815341723597 \quad 0.00774979970246 \mathrm{~h}$

$-3.32817925613785$

2.24526282945070

$-1.65512392902036 \mathrm{~h}$

7.43523359196750

$0.01283028185782 \mathrm{n}$

$-7.90360708971065$

5.03250614407513

-0.01297882273285 o

$-5.91301237190218$

1.29647980684953

-0.00732731654224 o

$-5.16314642228234$

8.05464051238878

$0.01188096374375 \mathrm{~h}$

Send 


\section{Coordinate Files for Optimized Geometries of Deprotonated Oxaloacetic Acid}<smiles>O=C(O)CC(=O)C(=O)[O-]</smiles>

$\begin{array}{lcccc}\text { Senergy SCF } & \text { SCFKIN } & \text { SCFPOT } & & \\ 1-530.0297541909 & 527.5074196714 & -1057.537173862 & \\ 45-530.1045271545 & 528.1354360043 & -1058.239963159 & \\ \text { \$end } & & & & \\ \text { \$coord } & & & & \\ -4.27925690580448 & -1.02487277213909 & -0.08767686213387 & \mathrm{c} \\ -1.88586851542744 & 0.57356900623240 & -0.19961144721638 & \mathrm{c} \\ 0.54112934754141 & -0.86131389741863 & 0.29506639304730 & \mathrm{c} \\ 3.03143546275298 & 0.56348177854812 & 0.25162433320910 & \mathrm{c} \\ 0.64219882163677 & -2.39692301252422 & -1.08380750120346 & \mathrm{~h} \\ 0.35806367912104 & -1.80367600875511 & 2.12434816962884 & \mathrm{~h} \\ 2.88471078525673 & 3.00082024754497 & -0.28399988828257 & \mathrm{o} \\ 5.01496239167232 & -0.47348395429110 & 0.67680522323942 & \mathrm{o} \\ -5.28512325440048 & -1.09915124764101 & 2.02517991359323 & \mathrm{o} \\ -4.77608978125121 & -2.07812850328982 & -2.12242826389612 & \mathrm{o} \\ 1.03848287495585 & 3.39329392224995 & -0.53711887901570 & \mathrm{~h} \\ -1.85931282580445 & 2.84592445663602 & -0.67051490219500 & \mathrm{o}\end{array}$

Send

\section{With COSMO}

Senergy SCF

$1-530.1352455991$

SCFKIN SCFPOT

$57-530.1996174736$

$527.4840650194 \quad-1057.619310619$

Send

$528.1444484406 \quad-1058.344065914$

\$coord

$\begin{array}{rrrl}-4.27443766867239 & -1.04827968043390 & -0.04382395900774 & \mathrm{c} \\ -1.86036730225126 & 0.51656354760708 & -0.18587252536262 & \mathrm{c} \\ 0.57080471005614 & -0.87438186599697 & 0.28318267894909 & \mathrm{c} \\ 3.01853656461352 & 0.60075196668801 & 0.23194757575507 & \mathrm{c} \\ 0.71089435627155 & -2.39728272402729 & -1.10979159226384 & \mathrm{~h} \\ 0.43341228838345 & -1.83826872198742 & 2.10692225302715 & \mathrm{~h} \\ 2.87248201961391 & 3.03013128990328 & -0.31203874319458 & \mathrm{o} \\ 5.02380920804457 & -0.40095516005268 & 0.66382913794745 & \mathrm{o} \\ -5.34305692195763 & -1.04857475669105 & 2.04658569080676 & \mathrm{o} \\ -4.85517731753431 & -2.13640280748665 & -2.04290047320715 & \text { o } \\ 1.05833608328486 & 3.46053184246479 & -0.57608083365605 & \mathrm{~h} \\ -1.92990393960336 & 2.77570708516518 & -0.67409292101872 & \mathrm{o}\end{array}$

\$end 
Coordinate Files for Optimized Geometries of Uncatalyzed Decarboxylation Transition

State<smiles>O=C1CC(C(=O)[O-])=CO1</smiles>

Without COSMO

$\begin{array}{lcccc}\text { \$energy SCF } & \text { SCFKIN } & \text { SCFPOT } & & \\ 1-530.0450625309 & 527.6086371147 & -1057.653699646 & \\ 62-530.0577409784 & 527.8465879239 & -1057.904328902 & \\ \text { \$end } & & & & \\ \text { \$coord } & & & & \\ -3.93293222139808 & -0.24748122384297 & 0.26635480020379 & \mathrm{c} \\ -1.17794198229385 & 0.37338054852618 & -0.30649059240391 & \mathrm{c} \\ 0.66260061953111 & -1.74802808036896 & -0.23749116320631 & \mathrm{c} \\ 4.13071418158366 & -0.40817810761207 & 0.44164999945593 & \mathrm{c} \\ 0.90412811389642 & -2.57679119162057 & -2.10771128125337 & \mathrm{~h} \\ 0.24852548554602 & -3.17993111991076 & 1.17039498026022 & \mathrm{~h} \\ 4.14073406888186 & 1.99967370097528 & 0.02976876698887 & \mathrm{o} \\ 5.81534935617802 & -1.75704847607465 & 1.05837969141626 & \mathrm{o} \\ -4.27948191074677 & -0.43484181708550 & 2.58184425723923 & \mathrm{o} \\ -5.33917938186392 & -0.53088503873973 & -1.57594733922257 & \mathrm{o} \\ 2.28466207268850 & 2.48846812265245 & -0.42198200554477 & \mathrm{~h} \\ -0.43887207395532 & 2.54319398357216 & -0.77155615885072 & \mathrm{o}\end{array}$

Send

With COSMO

Senergy SCF $1-530.1425614117$

SCFKIN SCFPOT

$71-530.1425628306$

$527.8549779781 \quad-1057.997539390$

Send

$527.8701542930 \quad-1058.012717124$

\$coord

$-3.69319402159054$

$-1.14917559810936$

$-0.49356695131291$

$0.22796841918397 \mathrm{c}$ 0.63111270631076 0.64593410566187

$-0.48524387114702 \mathrm{c}$ 4.02004571247071 $-0.84471748303088$ $-1.96918412737252 \mathrm{c}$ $-0.40493483932685$ $-0.04632768061470 \mathrm{c}$ 1.22620796954637 0.08733292058833

0.25446925162370 $-2.84647147802345$

4.00983237192440 1.79769599772889 $-3.70286330890529 \mathrm{~h}$ $-2.16717250772574 \mathrm{~h}$ 5.58590972270708 $-1.99830634353051$ 0.96505873183049 о $-3.56072929488055$ $-2.12704951111625$ -0.09783389516654 о 1.91597758563893 o $-5.56511204229214$ 0.31490504927729 -0.93518324931087 о 2.25573611804102 2.58206283089047 $-0.56578476596594$

2.78677978450797 $0.66702682731240 \mathrm{~h}$ 0.27280225915857 o

Send 
Coordinate Files for Optimized Geometries of Uncatalyzed Decarboxylation Intermediate<smiles>C=C(O)C(=O)[O-]</smiles>

Without COSMO

\begin{tabular}{lllll} 
Senergy SCF & SCFKIN & \multicolumn{2}{c}{ SCFPOT } & \\
$1-341.5897095674$ & 340.1266176292 & -681.7163271966 & \\
$13-341.6240598518$ & 340.3133673526 & -681.9374272044 & \\
\$end & & & & \\
\$coord & & & & \\
-4.34884724771373 & -2.93625653164959 & -0.09263753776147 & $\mathrm{c}$ \\
-2.17655424229989 & -0.99470702779651 & -0.01294304629221 & $\mathrm{c}$ \\
0.27819033969752 & -1.56307245545124 & -0.11661582949476 & $\mathrm{c}$ \\
0.80325716358594 & -3.52989426317681 & -0.26802055388390 & $\mathrm{~h}$ \\
1.72551975603691 & -0.11489614708325 & -0.05300309406167 & $\mathrm{~h}$ \\
-3.10564633222349 & 1.36069280992683 & 0.17631988305860 & $\mathrm{o}$ \\
-6.46876298147314 & -1.83655953471010 & 0.03713572612857 & $\mathrm{o}$ \\
-3.86490686562413 & -5.20765706157978 & -0.26233394300986 & $\mathrm{o}$ \\
-4.94535571423761 & 0.90916596910460 & 0.19168432501952 & $\mathrm{~h}$
\end{tabular}

\$end

With COSMO

Senergy SCF

$1-341.6995770143$

SCFKIN SCFPOT

$12-341.7224569113$

$340.1513421545 \quad-681.8509191688$

$340.3450131645-682.0674700759$

Send

\$coord

$\begin{array}{rrrl}-4.33396839036417 & -2.94614610061270 & -0.09372351904245 & \mathrm{c} \\ -2.19970449276889 & -1.01199087886447 & -0.01326569820195 & \mathrm{c} \\ 0.24970175578718 & -1.58008271179576 & -0.11716532613140 & \mathrm{c} \\ 0.80154499876326 & -3.53897431091672 & -0.26881906131436 & \mathrm{~h} \\ 1.69467954267732 & -0.13170104435523 & -0.05341666008015 & \mathrm{~h} \\ -3.08362559475712 & 1.37380452081466 & 0.17682317629093 & \mathrm{o} \\ -6.49284019444859 & -1.94832424612322 & 0.02955902675874 & \mathrm{o} \\ -3.82036239466945 & -5.22804408823156 & -0.26461675127167 & \mathrm{o} \\ -4.91853135447119 & 1.09827461766917 & 0.20421074269512 & \mathrm{~h}\end{array}$

\$end 
Coordinate Files for Optimized Geometries of Uncatalyzed Decarboxylation Product

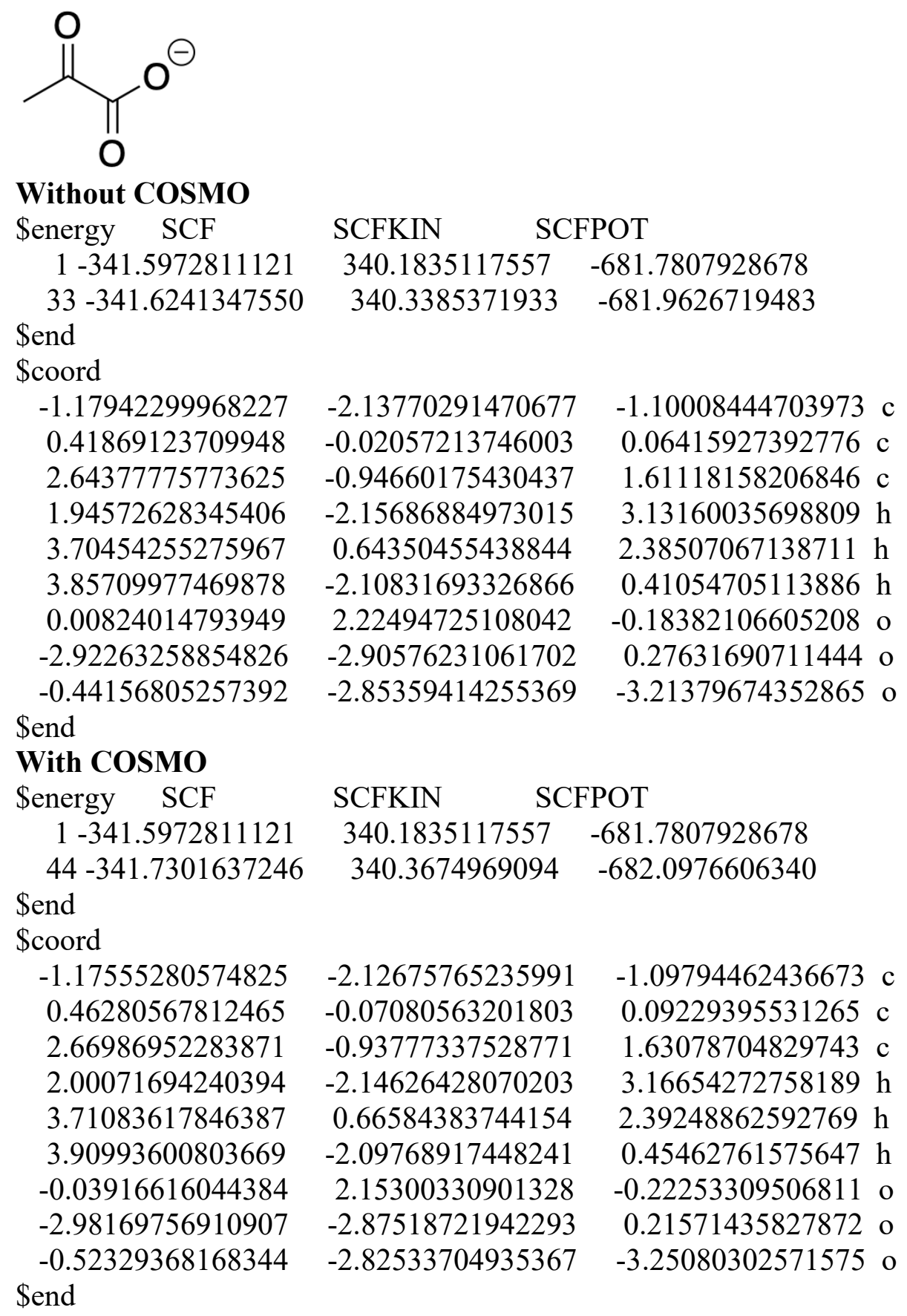




\begin{tabular}{|c|c|c|}
\hline Coordinate Files for $\mathbf{O}$ & otimized Geometries & of Carbon Dioxide \\
\hline Without COSMO & & \\
\hline Senergy SCF & SCFKIN & EPOT \\
\hline $1-188.4770559969$ & 187.6153602092 & -376.0924162061 \\
\hline $11-188.4792997339$ & 187.8206011469 & -376.2999008808 \\
\hline \$end & & \\
\hline \$coord & & \\
\hline-0.79870713486122 & 3.48581060521619 & $0.78908068363827 \mathrm{c}$ \\
\hline 1.05929405167456 & 4.62384666540040 & $1.17867975137030 \mathrm{o}$ \\
\hline-2.65670296143107 & 2.34776589490544 & $0.39948059429985 \mathrm{o}$ \\
\hline \$end & & \\
\hline With COSMO & & \\
\hline Senergy SCF & SCFKIN & FOT \\
\hline $1-188.4770559969$ & 187.6153602092 & -376.0924162061 \\
\hline $16-188.4821808758$ & 187.8116710016 & -376.2938518774 \\
\hline \$end & & \\
\hline \$coord & & \\
\hline-0.79870493454483 & 3.48580765735266 & $0.78908037269294 \mathrm{c}$ \\
\hline 1.05834231137275 & 4.62326586578329 & 1.178480569561380 \\
\hline-2.65575342144612 & 2.34834964238811 & 0.39968008705457 o \\
\hline \$end & & \\
\hline
\end{tabular}




\section{References}

(1) Kozlowski, J.; Zuman, P. Acid-Base, Hydration-Dehydration and Keto-Enol Equilibria in Aqueous Solutions of $\alpha$-Ketoacids: Study by Spectroscopy, Polarography and Linear Sweep Voltammetry. Bioelectrochemistry Bioenerg. 1992, 28 (1-2), 43-70.

https://doi.org/10.1016/0302-4598(92)80003-Y.

(2) Merz, J. H.; Waters, W. A. Some Oxidations Involving the Free Hydroxyl Radical. J. Chem. Soc. 1949, S15-S25.

(3) Monod, A.; Doussin, J. F. Structure-Activity Relationship for the Estimation of OHOxidation Rate Constants of Aliphatic Organic Compounds in the Aqueous Phase:

Alkanes, Alcohols, Organic Acids and Bases. Atmos. Environ. 2008, 42 (33), 7611-7622. https://doi.org/10.1016/j.atmosenv.2008.06.005.

(4) Doussin, J. F.; Monod, A. Structure-Activity Relationship for the Estimation of OHOxidation Rate Constants of Carbonyl Compounds in the Aqueous Phase. Atmos. Chem. Phys 2013, 13, 11625-11641. https://doi.org/10.5194/acp-13-11625-2013.

(5) Buxton, G. V; Greenstock, C. L.; Phillips Helman, W.; Ross, A. B.; Helman, W. P. Critical Review of Aqueous Solution Reaction Rate Constants for Hydrogen Atoms and Hydroxyl Radicals in Aqueous Solution. J. Phys. Chem. Ref. Data 1988, 17, 663. https://doi.org/10.1063/1.555805.

(6) Bruice, P. Y. Formation of a Carbinolamine Intermediate in the Tertiary Amine Catalyzed Enolization of Oxaloacetic Acid. an Alternative Mechanism for Enolization. J. Am. Chem. Soc 1983, 105, 4982-4996. 